\title{
Genetic ancestry of patients with porphyria cutanea tarda in a country with mixed races: a cross-sectional study (Rio de Janeiro - Brazil)*
}

\author{
Isabella Brasil Succi ${ }^{1}$ \\ Patricia Mariana Gonçalves da Rocha Porto Domingues ${ }^{3}$
}

\author{
Luís Cristóvão Pôrto ${ }^{2}$ \\ João Carlos Macedo Fonseca ${ }^{1}$
}

DOI: http:/ / dx.doi.org/10.1590/abd1806-4841.20186666

\begin{abstract}
Porphyria cutanea tarda has a complex etiology with genetic factors not completely elucidated. The miscegenation of the Brazilian population has important implications in the predisposition to diseases. There are no studies concerning the genetic ancestry of patients with porphyria cutanea tarda from a mixed population. Thirty patients living in Rio de Janeiro with sporadic porphyria cutanea tarda were studied for the genetic ancestry through informative markers - INDELS. There was a significant predominance of European ancestry across the sample of patients with porphyria cutanea tarda (70.2\%), and a small contribution of African and Amerindian ancestry, $20.1 \%$ and $10.9 \%$, respectively.
\end{abstract}

Keywords: Porphyria cutanea tarda; Genetics; Genomics; Population characteristics

Porphyria cutanea tarda (PCT) is the most frequent of all porphyrias and of greatest dermatological interest. It is classified as a chronic hepatic porphyria caused by the reduction in the activity of the enzyme uroporphyrinogen decarboxylase. The etiology is not completely clear, and environmental and genetic factors are needed for the clinical expression of the condition. ${ }^{1,2}$

It is known that the predisposition to different conditions varies considerably in the global population. However, since the Brazilian population went through an intense process of miscegenation, with contributions from Europeans, Africans and Amerindians, this resulted in a highly heterogenous genetic profile little seen in other parts of the world. ${ }^{3,4}$

Because of the Brazilian genetic diversity, despite having a higher frequency of reports of PCT in Caucasian patients, we do not know the potential role of the ancestry as an independent risk factor associated to the condition.

Currently, genetic ancestry has been applied in many populational studies and has a substantial advantage over physical features, since it remains constant throughout life. ${ }^{5}$
Ancestry-informative markers (AIMs) have a marked difference in the frequency of alleles among parental populations and are particularly useful to estimate individual ancestry proportions. In mixed populations, they are able to infer the genetic constitution that can result in important implications on the inherited diseases protein profile. . $^{5-7}$

We did not find in the literature data on the contribution of ancestry using AIMs in patients with PCT. To our knowledge, this is the first study that evaluated genetic ancestry in patients with PCT from a mixed population. Our aim is to investigate the influence of genetic origins to improve the understanding of the mechanisms involved in the predisposition to the disease.

Thirty patients from Rio de Janeiro, with clinical, laboratory and/or histopathologic diagnosis of sporadic PCT seen at the Hospital Universitário Pedro Ernesto/UERJ from 2012 to 2014 were included in the study.

All patients agreed to the consent form, that was signed by them and approved by the Ethics in Research Committee under the number CAAE: 17620613.0.0000.5259 and had blood samples taken

Received on 08.11.2016.

Approved by the Advisory Board and accepted for publication on 16.03.2017.

* Study conducted at Hospital Universitário Pedro Ernesto and Laboratory of Histocompatibility and Cryopreservation, Universidade do Estado do Rio de Janeiro (UERJ) - Rio de Janeiro (RJ), Brazil.

Financial support: This research project was partially funded by Rio de Janeiro Foundation Research Agency - FAPERJ and Brazilian National Council for Scientific and Technological Development -CNPq.

Conflict of interest: None.

Department of Dermatology, Hospital Universitário Pedro Ernesto - Universidade do Estado do Rio de Janeiro (UERJ) - Rio de Janeiro (RJ), Brazil.

Laboratory of Histocompatibility and Cryopreservation - Policlínica Piquet Carneiro - Universidade do Estado do Rio de Janeiro (UERJ) - Rio de Janeiro (RJ), Brazil.

Laboratory of DNA Diagnoses - Universidade do Estado do Rio de Janeiro (UERJ) - Rio de Janeiro (RJ), Brazil.

MAILING ADDRESS:

Isabella Brasil Succi

E-mail: ibsucci@unisys.com.br 


\begin{tabular}{|c|c|c|c|c|c|c|c|}
\hline Samples & $\%$ African & \%European & $\%$ Amerindian & Samples & $\%$ African & \%European & $\%$ Amerindian \\
\hline 1 & $31.8 \%$ & $45.8 \%$ & $22.4 \%$ & 16 & $38.0 \%$ & $49.0 \%$ & $13.0 \%$ \\
\hline 2 & $5.1 \%$ & $87.7 \%$ & $7.2 \%$ & 17 & $45.7 \%$ & $47.7 \%$ & $6.6 \%$ \\
\hline 3 & $3.1 \%$ & $93.7 \%$ & $3.3 \%$ & 18 & $16.4 \%$ & $77.1 \%$ & $6.5 \%$ \\
\hline 4 & $16.2 \%$ & $81.1 \%$ & $2.7 \%$ & 19 & $44.5 \%$ & $47.6 \%$ & $7.9 \%$ \\
\hline 5 & $47.8 \%$ & $44.7 \%$ & $7.6 \%$ & 20 & $8.6 \%$ & $81.0 \%$ & $10.4 \%$ \\
\hline 6 & $21.7 \%$ & $70.2 \%$ & $8.2 \%$ & 21 & $27.0 \%$ & $67.5 \%$ & $5.4 \%$ \\
\hline 7 & $16.0 \%$ & $74.6 \%$ & $9.4 \%$ & 22 & $5.5 \%$ & $86.8 \%$ & $7.7 \%$ \\
\hline 8 & $13.2 \%$ & $63.8 \%$ & $23.0 \%$ & 23 & $9.8 \%$ & $84.6 \%$ & $5.6 \%$ \\
\hline 9 & $25.0 \%$ & $69.3 \%$ & $5.7 \%$ & 24 & $11.6 \%$ & $73.6 \%$ & $14.8 \%$ \\
\hline 10 & $15.8 \%$ & $74.6 \%$ & $9.6 \%$ & 25 & $11.3 \%$ & $74.2 \%$ & $14.5 \%$ \\
\hline 11 & $13.9 \%$ & $77.8 \%$ & $8.3 \%$ & 26 & $3.4 \%$ & $93.3 \%$ & $3.3 \%$ \\
\hline 12 & $14.9 \%$ & $76.0 \%$ & $9.1 \%$ & 27 & $4.3 \%$ & $87.1 \%$ & $8.6 \%$ \\
\hline 13 & $7.5 \%$ & $82.8 \%$ & $9.7 \%$ & 28 & $24.0 \%$ & $69.3 \%$ & $6.7 \%$ \\
\hline 14 & $25.8 \%$ & $66.9 \%$ & $7.3 \%$ & 29 & $65.6 \%$ & $29.1 \%$ & $5.3 \%$ \\
\hline 15 & $9.3 \%$ & $53.9 \%$ & $36.9 \%$ & 30 & $20.6 \%$ & $75.4 \%$ & $4.0 \%$ \\
\hline
\end{tabular}

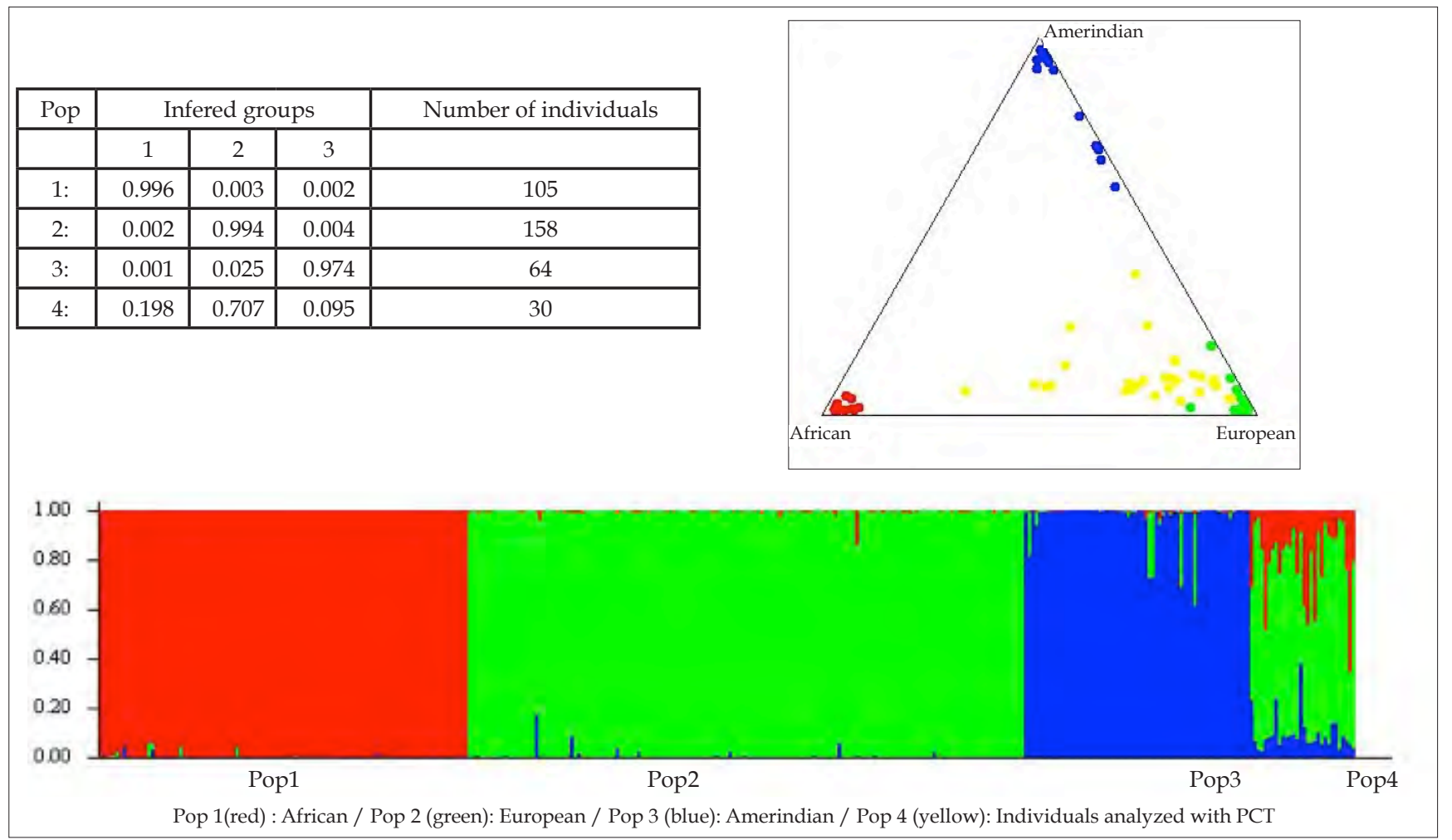

FIGURE 1: Distribution of the patients with PCT regarding ethnic mixture with Europeans, Africans and Amerindians

for the analysis of genetic ancestry. Each genetic ancestry was determined by AIMs.

A panel with 46 ancestry-informative markers - INDELS, which has allelic frequencies with significant differences between Europeans, Africans and native Americans, was genotyped in one single PCR multiplex followed by capillary electrophoresis, according to the protocol described by Pereira et al. ${ }^{8}$ Amplified fragments marked with dye were separated and detected by the automatic se- quencer ABI 3500 (Life Technologies). The samples were genotyped using the program GeneMapper v.4.1 (Life Technologies). The nomenclature of the alleles is according to Pereira et al. ${ }^{8}$

To test the population structure and estimate the proportions of ancestry, the Structure software v2.3.3 was used. In this study, considering the historical development of the Brazilian population, we assumed an essentially tri-hybrid contribution of native Americans, Europeans and Africans (ex, $\mathrm{K}=3$ ) for the current genetic 
composition of the Brazilian population.

AIMs were used in the study patients to estimate the proportion of African, European and native American ancestry. The results of the analysis are presented in table 1 and figure 1.

We calculated the mean percentage of the 30 patients with PCT, according to the genetic ancestry obtained by the AIMs. There was a significant predominance of European ancestry $(70.2 \%)$ and a small contribution from the African and native American origins (20.1\% and $10.9 \%$, respectively).

Analyzing the median, we observed that half of the sample had a percentage of European contribution higher than $74.4 \%$. For the African and Amerindian ancestries, the values were $15.9 \%$ and $7.8 \%$, respectively.

This datum supports the fact that most of the studies of PCT groups come from homogenous populations, mainly European, such as Hungary, Sweden, Norway, and so forth ${ }^{2,9}$, as well as the mutation $\mathrm{C} 282 \mathrm{Y}$ in the hereditary hemochromatosis gene, frequent in European descendants, which was also observed in PCT patients, contributing to an iron overload. ${ }^{9}$
Manta et al. ${ }^{10}$ evaluated the proportion of ethnic mix in individuals born in Rio de Janeiro using autosomal markers. In this study, a predominantly European influence was seen (55.2\%), with a small contribution from the African (31.1\%) and Amerindian $(13.7 \%)$ origins.

Comparing the genetic ancestry only of people born in Rio de Janeiro (23 patients) with the study conducted by Manta et al., ${ }^{10}$ we observed that 17 (73.91\%) of the 23 patients had a proportion of European ancestry above the $55.2 \%$ found in this study. In the case of African ancestry, we had $5(21.74 \%)$ patients with a percentage above the estimate by Manta et al.; and for Amerindian, the percentage was of $17.39 \%$ (4 patients) (Chi-square test (P-value): 0.000).

Therefore, in areas with a higher European ancestry, it would be interesting to organize the health system and incorporate specific diagnostic tests and improving therapeutic guidelines to include PCT.

In sum, in our PCT patient sample from a mixed population, distribution data according to genetic ancestry showed a significant predominance of contribution of European origin. $\square$

\section{REFERENCES}

1. Schulenburg-Brand D, Katugampola R, Anstey AV, Badminton MN. The cutaneous porphyrias. Dermatol Clin. 2014;32:369-84, ix.

2. Horner ME, Alikhan A, Tintle S, Tortorelli S, Davis DM, Hand JL. Cutaneous porphyrias part 1: epidemiology, pathogenesis, presentation, diagnosis and histopathology. Int J Dermatol. 2013;52:1464-80.

3. Neto VVG, Santos RV. Biorrevelações: testes de ancestralidade genética em perspectiva antropológica comparada. Horiz Antropol. 2011;35:227-55.

4. Ramos BR, D'Elia MP, Amador MA, Santos NP, Santos SE, da Cruz Castelli E, et al. Neither self-reported ethnicity nor declared family origin are reliable indicators of genomic ancestry. Genetica. 2016;144:259-65.

5. Cardena MM, Ribeiro-Dos-Santos A, Santos S, Mansur AJ, Pereira AC, Fridman $\mathrm{C}$, et al. Assessment of the relationship between self-declared ethnicity, mitochondrial haplogroups and genomic ancestry in brazilian individuals. PLoS One. 2013;8:e62005.
6. Leite TK, Fonseca RM, de França NM, Parra EJ, Pereira RW. Genomic ancestry, self-reported "color" and quantitative measures of skin pigmentation in Brazilian admixed siblings. PLoS One. 2011;6:e27162.

7. Cavalcante LN, Stefano JT, Machado MV, Mazo DF, Rabelo F, Sandes KA, et al. Genetic ancestry in non-alcoholic fatty liver disease patients from Brazil and Portugal. World J Hepatol. 2015;7:1433-8.

8. Pereira R, Phillips C, Pinto N, Santos C, dos Santos SE, Amorim A, et al. Straightforward inference of ancestry and admixture proportions through ancestry-informative insertion deletion multiplexing. PLoS One. 2012;7:e29684.

9. Nagy Z, Kószó F, Pár A, Emri G, Horkay I, Horányi M, et al. Hemochromatosis (HFE) gene mutations and hepatitis $C$ virus infection as risk factors for porphyria cutanea tarda in Hungarian patients. Liver Int. 2004;24:16-20.

10. Manta FS, Pereira R, Caiafa A, Silva DA, Gusmão L, Carvalho EF. Analysis of genetic ancestry in the admixed Brazilian population from Rio de Janeiro using 46 autosomal ancestry-informative indel markers. Ann Hum Biol. 2013:40:94-8.

How to cite this article: Succi IB, Pôrto LC, Domingues PMGRP, Fonseca JCM. Genetic ancestry of patients with porphyria cutanea tarda in a country with mixed races: a cross-sectional study (Rio de Janeiro - Brazil). An Bras Dermatol. 2018;93(1):148-50. 